\title{
Evaluación de la biomasa residual (cereza) de café como sustrato para el cultivo del hongo comestible Pleurotus ostreatus
}

\author{
David E. González; Juan E. Ortiz; Yisel Mejía; Luz S. García-Alzate; Ximena Cifuentes-Wchima \\ Universidad La Gran Colombia, Facultad de Ingenierías, Grupo de Investigación Gerencia de la Tierra, Campus \\ Ciudadela del Saber La Santa María, km 7 vía Armenia - La Tebaida. \\ *garciaalzluz@miugca.edu.co
}

Fecha recepción: octubre 15 de 2019 Fecha aceptación: enero 20 de 2020

\begin{abstract}
Resumen
Colombia es un país productor de café y se estima que el $60 \%$ son residuos, donde la cereza es uno de ellos; este material orgánico al entrar en descomposición puede ser dañino para la población, el ambiente y los cultivos aledaños. El objetivo de esta investigación fue evaluar la biomasa residual (cereza) de café como sustrato para el cultivo del hongo comestible Pleurotus ostreatus, con el fin de otorgar un mayor valor agregado a estos residuos. La biomasa residual (cereza) de café fue suministrada por agricultores del departamento del Quindío, la cual se caracterizó fisicoquímicamente, analizando parámetros como $\mathrm{pH}$, humedad, fibra, grasa, celulosa, lignina, cenizas, materia orgánica y nitrógeno, para luego ser empleada como sustrato en el cultivo del hongo y finalmente establecer si se puede emplear como medida de reducción de residuos. La caracterización fisicoquímica de la biomasa reportó pH del 6,693 $\pm 0,041$, porcentaje de humedad del $82,875 \pm 0,209$, contenido de lignina y celulosa del 28,954 $\pm 0,046$ y 48,390 $\pm 0,326$, respectivamente y materia orgánica del 13,249 $\pm 0,187$. En el cultivo del hongo, se observó la aparición de los primordios a los 15 días y el hongo para cosechar a los 20 días, reduciendo el volumen de residuos en un 80 \%. Para concluir, la caracterización fisicoquímica del residuo reportó componentes adecuados para emplearlo como sustrato en el cultivo de hongos Pleurotus ostreatus, siendo importante que estos residuos sean empleados para la generación de un nuevo producto y/o la disminución de este, lo que lograría un equilibrio entre el ambiente y los residuos que se generan.
\end{abstract}

Palabras clave: Biomasa; Café; Caracterización; Cultivo; Residuos. 


\title{
Evaluation of the residual biomass (cherry) of coffee as a substrate for the cultivation of the edible fungi Pleurotus ostreatus
}

\begin{abstract}
Colombia is a coffee producing country and it is estimated that $60 \%$ are residues, where cherry is one of them; this organic material when decomposing can be harmful to the population, the environment and surrounding crops. The objective of this investigation was to evaluate the residual biomass (cherry) of coffee as a substrate for the cultivation of edible fungus Pleurotus ostreatus, in order to give greater added value to these residues. The residual biomass (cherry) of coffee was supplied by farmers in the department of Quindío, which was characterized physicochemical, analyzing parameters such as $\mathrm{pH}$, humidity, fiber, fat, cellulose, lignin, ashes, organic matter and nitrogen; to then be used as a substrate in the cultivation of the fungus and finally establish if it can be used as a measure of waste reduction. The physicochemical characterization of biomass reported $\mathrm{pH}$ of $6.693 \pm 0.041$, humidity percentage of $82.875 \pm 0.209$, lignin and cellulose content of $28.954 \pm 0.046$ and 48.390 \pm 0.326 , respectively and organic matter of $13.249 \pm 0.187$. In the cultivation of the fungus, the appearance of the primordia was observed at 15 days and the fungus to harvest at 20 days, reducing the volume of waste by $80 \%$. To conclude, the physicochemical characterization of the residue reported adequate components to be used as a substrate in the cultivation of Pleurotus ostreatus fungi, it is important that these wastes are used for the generation of a new product and/or the reduction of this, which would achieve a balance between the environment and the waste that is generated.
\end{abstract}

Keywords: Biomass; Coffee; Characterization; Cultivation; Waste.

\section{Avaliação da biomassa residual (cereja) do café como substrato para o cultivo do fungo comestível Pleurotus ostreatus}

\begin{abstract}
Resumo
A Colômbia é um país produtor de café e estima-se que 60 \% sejam resíduos, onde a cereja é um deles; esse material orgânico ao se decompor pode ser prejudicial à população, ao meio ambiente e às cultivos vizinhas. O objetivo desta investigação foi avaliar a biomassa residual (cereja) do café como substrato para o cultivo do fungo comestível Pleurotus ostreatus, a fim de agregar maior valor agregado a esses resíduos. A biomassa residual (cereja) do café foi fornecida pelos agricultores do departamento de Quindío, que foi caracterizada físico-quimicamente, analisando parâmetros como pH, umidade, fibra, graxa, celulose, lignina, cinzas, matéria orgânica e nitrogênio; para despois ser usado como substrato no cultivo do fungo e finalmente estabelecer se ele pode ser usado como medida de redução de resíduos. A caracterização físico-química da biomassa reportou $\mathrm{pH} 6,693 \pm 0,041$, porcentagem de umidade de 82,875 $\pm 0,209$, teor de lignina e celulose de $28,954 \pm 0,046$ e 48,390 $\pm 0,326$, respectivamente, e matéria orgânica de 13,249 $\pm 0,187$. No cultivo do fungo, observou-se o aparecimento dos primórdios aos 15 dias e o fungo colhendo aos 20 dias, reduzindo o volume de resíduos em 80 \%. Concluindo, a caracterização físico-química do resíduo relatou componentes adequados para serem utilizados como substrato no cultivo de fungos Pleurotus ostreatus, é importante que esses resíduos sejam utilizados para a geração de um novo produto e/ou a redução deste, o que alcançaria um equilíbrio entre o meio ambiente e os resíduos gerados.
\end{abstract}

Palavras-chave: Biomassa; Café; Caracterização; Cultivo; Resíduos. 


\section{Introducción}

En la actualidad, una de las fuentes de ingreso más fuertes de manera económica en Colombia se deriva de la explotación de la tierra en sus diversas formas; una de esas es la agricultura, siendo uno de los sectores de mayor envergadura en el país, representando cerca del $4 \%$ del producto interno bruto [1]. Colombia ha sido y sigue siendo uno de los países con mayor producción de café, generando cerca de 14 millones de sacos de café de $60 \mathrm{~kg}$ por año, ubicándose así en el tercer lugar a nivel mundial con mayor exportación de este grano, según lo reportado en [2] y [3]. De manera más regional, la agricultura en el departamento del Quindío predomina por cerca del $52 \%$ del territorio total, encabezado por diversos cultivos, como lo son el plátano, el banano, el cacao, los cítricos y, principalmente, el café [4].

Dentro de los componentes con los que cuenta el café están la pulpa y el mucílago; estas biomasas constituyen los subproductos más abundantes del proceso de beneficio húmedo del café y representan alrededor del $60 \%$ del peso del fruto fresco, lo que indica que casi la mitad son desechos orgánicos [5]. La biomasa cubre un amplio espectro de materiales orgánicos, y por tal motivo, es considerada una mezcla compleja de polímeros de carbohidratos conocidos como celulosa, hemicelulosa, lignina y pequeñas cantidades de otras sustancias contenidas en la pared celular de las plantas [6]. La naturaleza y la composición de los productos obtenidos a partir del empleo de la biomasa dependen de la composición química del tipo de biomasa tratada y de las condiciones de operación, como lo describen Akhtar y NorAishah Saidina [7].

Una de las grandes problemáticas que presenta el territorio colombiano y la mayoría de los países agrícolas, es el poco aprovechamiento que se le da a los residuos orgánicos, ya que la gran mayoría son utilizados como abonos naturales, lo cual tiene sus beneficios y sus perjuicios, ya que esta materia orgánica al entrar en descomposición puede generar lixiviados que pueden ser ricos en metales y, a su vez, nocivos para los cultivos aledaños [8].

El grano de café se encuentra conformado por seis distintas partes (epicarpio, disco, mesocarpio, endocarpio, película plateada y el embrión), en donde la parte exterior del fruto es el pericarpio o más conocido como la cereza del café [9], como se muestra en la figura 1. En la presente investigación, la cereza fue el componente más importante y la materia prima, ya que a partir de esta biomasa residual se pretendía producir hongos comestibles.

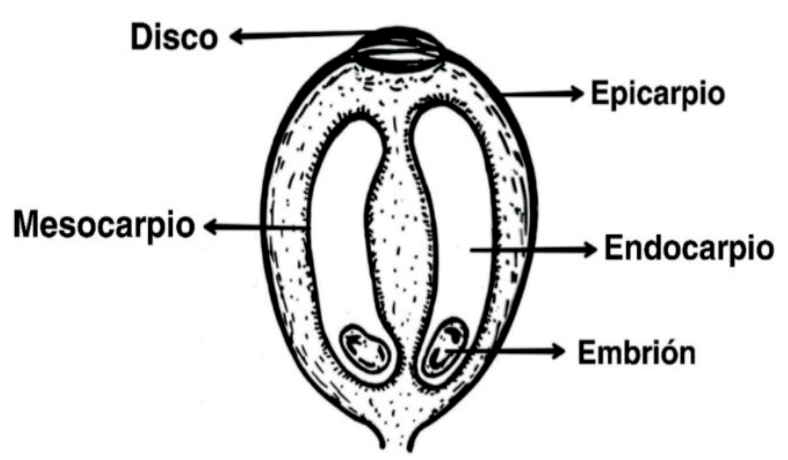

Figura 1. Partes del café. Fuente: autores.

Ahora bien, es necesario mencionar que estos desechos pueden ser utilizados de otra manera, teniendo como resultado un aprovechamiento más alto de lo que se genera después de la cosecha y así se podría disminuir la gran cantidad de residuos que se tienen; además de esto, se lograría un equilibrio entre el ambiente y los desechos que se generan.

El crecimiento de la población, la industrialización y la urbanización han generado como resultado la generación de desechos sólidos y líquidos. La gestión de estos residuos es un problema mundial, ya que su utilización es limitada; es por esto que se deben buscar alternativas de manejo de estos desechos, donde la disposición final de este material sea diferente a la que comúnmente se tiene, como lo expresan Rathinavelu, Giorgio [10] y Rodríguez [11]. Por lo tanto, de acuerdo a la caracterización de esta biomasa residual (cereza de café) se pudo establecer su posible empleo como sustrato para la obtención de cuerpo fructífero Pleurotus ostreatus mediante la degradación orgánica de esta biomasa, que permitiría disminuir volúmenes de residuos y generar un subproducto de esta, generando una biorremediación ambiental (micorremediación); estos hongos no sólo secretan celulosa y hemicelulosas, sino también tienen la capacidad de producir enzimas que degradan la lignina, siendo los mejores para este procedimiento biológico.

Al tener los residuos orgánicos y dejarlos al contacto con el terreno, se originan gases como el $\mathrm{CO}_{2}$ que, al estar en el ambiente junto a las crecientes emanaciones de otros gases efectos invernaderos, van calentando gradualmente el 
planeta, elevando la temperatura del sistema atmosférico, tal y como lo reporta Alvares Torres [12], quien describe algunas de las problemáticas generadas en la ciudad de Bogotá, y por lo tanto las posibles afectaciones al medio. Además de esto, la acumulación de este biomasa residual (cereza de café) puede ocasionar proliferación de algunos insectos y roedores que a su vez derivan problemas en la salud de los campesinos y en los cultivos aledaños al sitio de acopio de esta biomasa. Es por lo anteriormente mencionado que aprovechar la biomasa residual para la producción de cuerpos fructíferos (hongos macromicetos) es una alternativa viable, debido a que se reducen en gran porcentaje estos residuos que se han generado después de la posproducción, siendo una alternativa verde.

El objetivo de esta investigación fue evaluar la biomasa residual (cereza) de café como sustrato para el cultivo del hongo comestible Pleurotus ostreatus, con el fin de otorgar un mayor valor agregado a estos residuos y así reducir su impacto ambiental.

\section{Metodología}

La metodología propuesta en esta investigación se realizó en varias etapas desde la obtención de la biomasa residual, a la cual se le realizó la caracterización fisicoquímica, para posterior cultivo del hongo Pleurotus ostreatus; como se muestra a continuación.

\section{Obtención de biomasa residual}

La biomasa residual utilizada en esta investigación (cereza de café) fue suministrada por productores del departamento del Quindío, siendo este un café de variedad especial, la cual fue entregada a los investigadores luego del debido procesamiento del grano en las fincas cafeteras de la región ubicadas en los municipios de Pijao, Montenegro y Circasia, quienes se predisponían a desechar esta biomasa en las zonas aledañas de los cultivos.

Las respectivas determinaciones fisicoquímicas se llevaron a cabo en los laboratorios del Campus Ciudadela del Saber La Santa María, Universidad La Gran Colombia.

\section{Caracterización fisicoquímica de la biomasa residual \\ En esta fase se emplearon diferentes normas y protocolos para cada evaluación. Para la determinación de $\mathrm{pH}$ se empleó potenciómetro}

teniendo en cuenta el método AOAC 981.12 [13] [13]. La determinación de porcentaje de humedad se realizó con el uso de una estufa de convección forzada ESCO bajo los parámetros establecidos en el método AOAC 20.013 [14]. El porcentaje de nitrógeno se determinó por medio del método Kjeldahl acuerdo a lo señalado por la norma NTC 4657 [15]. La determinación del porcentaje de cenizas se llevó a cabo mediante gravimetría con calcinación en mufla TERRÍGENO de acuerdo a la norma TAPPI 211 om-02 [16]. El porcentaje de materia orgánica se calculó empleando la norma mexicana NMX-AA-21 [17]; las determinaciones del porcentaje de grasa y fibra se realizaron por el método de extracción Soxhlet, de acuerdo a la metodología establecida en las norma NTC 1142 [18] y con el método AOAC 962.06 [19], respectivamente, el porcentaje de lignina con la norma TAPPI 222 om-88 [20] y finalmente, la determinación del porcentaje de celulosa con lo establecido por el método NTC 697 [21].

\section{Cultivo del hongo Pleurotus ostreatus}

Condiciones físicas del cultivo. Posteriormente a la obtención de la biomasa residual, se establecieron las condiciones a tener en cuenta durante la realización del cultivo, los cuales fueron un porcentaje de humedad relativa (\%HR), temperatura ambiente $\left({ }^{\circ} \mathrm{C}\right.$ ) y luminosidad (Lux). El cultivo del hongo fue realizado en un cobertizo de construcción liviana situado en las coordenadas Norte $4^{\circ} 32^{\prime} 14^{\prime \prime}$ y $75^{\circ} 40^{\prime} 02^{\prime \prime}$ Oeste en la ciudad de Armenia, Quindío, como se observa en la figura 2.

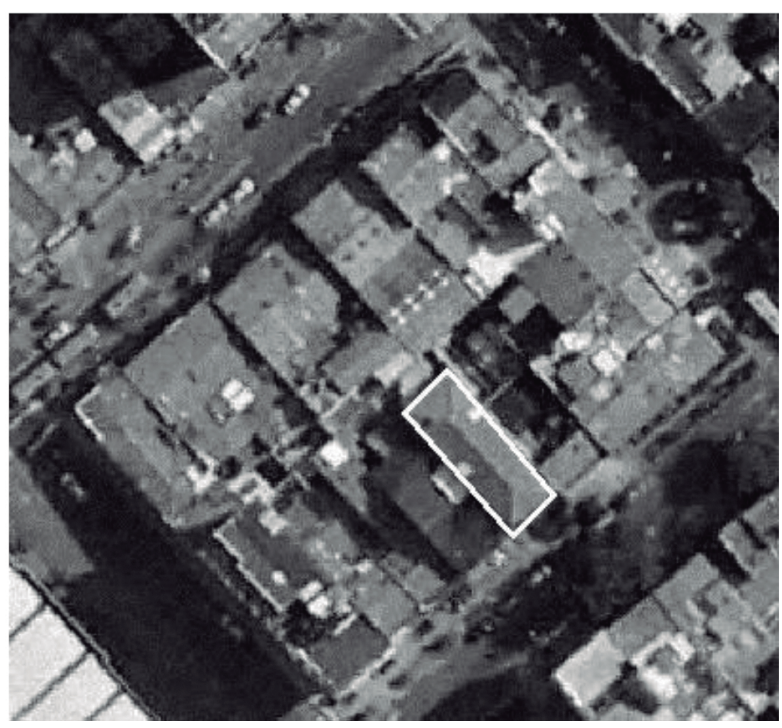

Figura 2. Localización geográfica del cultivo del hongo [4]. 
Obtención de hongos Pleurotus ostreatus Para la obtención del hongo cultivado en la biomasa residual de café se realizaron varios procesos como lo fueron: el acondicionamiento del sustrato, la inoculación e incubación de la semilla y, finalmente, la fructificación y cosecha, teniendo en cuenta diferentes literaturas como se muestra a continuación:

Acondicionamiento del sustrato. Previo a la realización de la siembra de la semilla, la muestra fue desinfectada a temperatura ambiente por inmersión en una solución de carbonato de calcio al $0,6 \%$ durante un periodo total de 12 días, con lavados diarios [22].

Inoculación e incubación. Posterior al acondicionamiento del sustrato, se efectuó la inoculación en condiciones óptimas de asepsia mezclando sustrato con semilla a una concentración del $4 \%$. En donde se empacaron bolsas con un peso total que variaba entre los 1200 y 1500 gramos.Durante el tiempo de incubación, se realizó monitoreo de variables como temperatura y humedad relativa en rangos entre $22-24{ }^{\circ} \mathrm{C}$ y $70-85 \%$, respectivamente, además de tener la menor cantidad de iluminación posible y una baja ventilación para así obtener el crecimiento del hongo y garantizar las condiciones del cultivo, pues en esta fase se busca que el micelio pueda invadir totalmente el sustrato. Para poder obtener el porcentaje de humedad, se realizaron riegos a diferentes horas del día, generando así el ambiente óptimo para el debido desarrollo de la seta [22].

Fructificación y cosecha.Terminado el proceso de incubación, se procedió a trasladar las bolsas al área de producción en donde se situaron a la luz natural, para así inducir el crecimiento y formación de la seta. Finalmente, se llevó a cabo la cosecha del cuerpo fructífero de manera manual, en donde se adaptó un sitio con el fin de cumplir con las indicaciones establecidas por Rodríguez Valencia et al. [23] y Gaitán et al. [24] y extraer el hongo cuando el píleo estaba casi plano, de acuerdo a lo establecido por García Alzate et al. [22].

\section{Análisis estadístico}

Se realizó un estudio de la significancia de los diferentes efectos y comportamiento de las diferentes variables mediante un análisis de la varianza (ANOVA) para efectos aleatorio, usando el método LSD (least square differences) con un nivel de significancia $(\alpha)$ de 0,05 , soportado en el paquete estadístico STATGRAPHICS Centurión XV (Versión 15.2.05). Lo anterior, se hizo para las variables de los análisis fisicoquímicos de la biomasa residual (cereza de café) y la cantidad obtenida de cuerpo fructífero del cultivo.

\section{Resultados y discusión}

La caracterización fisicoquímica de la cereza de café muestra el potencial para generar alternativas de uso agroindustrial; esto se puede deber a la naturaleza del tejido, lo cual se evidencia en el perfil composicional, como se muestra en la tabla 1.

En la tabla 1 se evidencia que la biomasa residual cuenta con variables que le dan un valor agregado a este tipo de material que puede ser empleado con diferentes fines, como lo son la obtención de azúcares más simples, absorción de metales, la producción de bioetanol a través de la transformación de la celulosa, entre otros procesos.

Tabla 1. Caracterización de la biomasa residual (cereza de café).

\begin{tabular}{cc}
\hline $\begin{array}{c}\text { Variable } \\
( \pm \mathbf{D} . \mathbf{S})\end{array}$ & $\begin{array}{c}\text { Biomasa } \\
\text { (Cereza de café) }\end{array}$ \\
\hline $\mathrm{pH}$ & $6,693 \pm 0,041$ \\
Humedad (\%) & $82,875 \pm 0,209$ \\
Cenizas (\% bs) & $3,876 \pm 0,125$ \\
Mat. Orgánica (\% bs) & $13,249 \pm 0,187$ \\
Nitrógeno (\% bs) & $2,327 \pm 0,024$ \\
Grasa (\% bs) & $2,503 \pm 0,137$ \\
Fibra (\% bs) & $16,182 \pm 0,115$ \\
Lignina (\% bs) & $28,954 \pm 0,046$ \\
Celulosa (\% bs) & $48,390 \pm 0,326$ \\
\hline
\end{tabular}

n:3. ( \pm D. S): desviación estándar. bs: base seca.

Al observar los resultados que arrojó la caracterización fisicoquímica del material vegetal, se puede concluir que la variable $\mathrm{pH}$ con valores de 6,693 \pm 0,041 fue similar con lo establecido por García Alzate et al. [22] quienes trabajaron sobre sustratos de residuos orgánicos domésticos para la producción de hongos y autores como Miles y Chang [25] quienes establecieron los valores ideales de $\mathrm{pH}$ en sustratos para su posterior uso en cultivos de hongo Pleurotus ostreatus, siendo el rango de $\mathrm{pH}$ adecuado entre 4,0 y 8,0 .

Se obtuvo un valor de $82,875 \pm 0,209 \%$, para el contenido de humedad, lo cual establece que el sustrato cuenta con la capacidad de permitirle al 
hongo realizar los procesos químicos y bioquímicos que apoyen su metabolismo, generando así una invasión del micelio y desarrollo de los primordios, de acuerdo con lo reportado por Miles y Chang [25], quienes identificaron la influencia de este tipo de variables para el crecimiento de microorganismos. En cuanto al porcentaje de cenizas, este arrojó un valor $3,876 \pm 0,125 \%$, con lo cual se puede establecer que este posible sustrato cuenta con macro y micronutrientes inorgánicos que pueden ser adecuados para el crecimiento del hongo, tal como lo reportan Grau et al. [26].

El porcentaje de materia orgánica que se obtuvo fue de $13,249 \pm 0,187 \%$, el cual indica que la cereza de café cuenta con un potencial de almacenamiento de agua, facilita la absorción de nutrientes esenciales para el desarrollo del hongo Pleurotus ostreatus, según lo reportado por Siguas [27]. El porcentaje de nitrógeno en esta investigación arrojó un valor de $2,327 \pm 0,024 \%$, con el cual, al ser comparado con lo escrito por Docampo [28], se puede establecer que el cuerpo fructífero del hongo va a desarrollarse en buenas condiciones, debido a que el primordio puede aprovechar la disponibilidad de esta sustancia química que contiene la biomasa residual, en este caso la cereza del café, para la producción de cultivos. Autores como Moran et al. [29] indican que la materia orgánica al estar presente en el sustrato facilita el transporte de nutrientes, incrementa la actividad microbiana, y permite mayor retención de agua, lo que genera sistemas biológicos de mejor tamaño, con características físicas, químicas y biológicas adecuadas.

Por otra parte, en cuanto a las determinaciones de grasa y fibra, estas reportaron un porcentaje de $2,503 \pm 0,137$ y $16,182 \pm 0,115 \%$, respectivamente, con los cuales, al compararse con los resultados reportados por Toledo [30], se establece una similitud en las variables anteriormente mencionadas; estos componentes presentes en una matriz vegetal como es la cereza de café permite ser considerados a la hora de realizar formulación de otro tipo de subproducto derivado, como lo puede ser un lombricompost o un abono orgánico.

Finamente, el porcentaje de lignina presente en el material vegetal corresponde a un valor de alrededor del $28,954 \pm 0,046 \%$, siendo este un polímero orgánico requerido como fuente para el desarrollo de cuerpos fructíferos, como lo establecen García Alzate et al. [22], los cuales trabajaron con residuos orgánicos domésticos, indicando la importancia de este componente para permitir el desarrollo de hongos Pleurotus ostreatus. En cuanto al porcentaje de celulosa obtenido en esta investigación, este arrojó un valor de $48,390 \pm 0,326 \%$, y esta biomolécula orgánica es vital para el crecimiento de hongos del género Pleurotus, como lo mencionan en sus investigaciones autores como García Alzate et al. [22] y Miles y Chang [25]; además, autores como Blandón et al. [31] indican que este componente debe encontrarse en un rango entre 40 y $60 \%$, donde el sustrato utilizado cuente con la capacidad de brindarle esta sustancia al hongo, permitiendo la invasión del micelio y crecimiento del cuerpo fructífero. Las variables lignina y celulosa determinadas en esta investigación fueron las más relevantes, esto debido a que estructuras biológicas como el hongo Pleurotus ostreatus son setas lignocelulósicas, lo que indica que se alimentan principalmente de estos biopolímeros (lignina y celulosa), como lo indican Alzate et al. [22], Miles y Chang [25] y Michel et al. [32].

Los resultados mostrados en esta caracterización fisicoquímica son necesarios para indicar posibles usos posteriores a este tipo de residuos vegetales; autores como Alzate et al. [22], Moran et al. [29] y Song [33] trabajaron con diferentes sustratos como lo son los residuos orgánicos domésticos y sustratos de maíz y quinua para la producción de setas comestibles, haciendo referencia a la importancia de las variables de humedad, lignina y celulosa como condiciones para el crecimiento y desarrollo del cuerpo fructífero; lograron establecer en esta investigación que la biomasa residual cereza de café posiblemente puede ser usada para este fin.

Es por esto que es de suma importancia mencionar que la composición de esta biomasa residual cumple con las características necesarias para la producción de hongos comestibles, tal y como lo es el Pleurotus ostreatus, pero también que, de esta manera, se contribuye a la disminución de los impactos ambientales que son generados debido a los grandes volúmenes de este residuo agroindustrial, ya que por medio de esta investigación se pretende reducir la acumulación de esta materia orgánica [34].

En la tabla 2 y figura 3 se muestran los resultados obtenidos en el cultivo del hongo Pleurotus ostreatus sobre el sustrato de cereza de café; la tabla 2 presenta las condiciones de humedad relativa $(\% \mathrm{HR})$, temperatura $\left({ }^{\circ} \mathrm{C}\right)$ y luz (lux) establecidas durante las tres cosechas del cultivo y la figura 3 muestra la obtención del hongo Pleurotus ostreatus cultivado en biomasa residual cereza de café.

Como se puede observar en la tabla 2, se obtuvieron los valores de humedad relativa $(\% \mathrm{RH})$, temperatura 
$\left({ }^{\circ} \mathrm{C}\right)$ y luminosidad (Lux), durante las tres cosechas del cultivo; los resultados arrojaron rangos entre $73,9 \pm 8,5$ y $83,7 \pm 4,6$ para la humedad relativa, entre $22,4 \pm 1,6$ y $23,5 \pm 0,9$ para la temperatura y entre $292,3 \pm 0,021^{\circ} \mathrm{C}$ y $293,2 \pm 0,015$ Lux para la luminosidad. Estos resultados nos permitieron establecer las condiciones en las cuales se encontraba el cultivo para un óptimo desarrollo del hongo, siendo similar a lo establecido por Michel et al. [32], con valores reportados de humedad relativa $(70-90 \% \mathrm{RH})$ y de intensidad de luz (290 - 295 lux), con los cuales se logra el desarrollo del hongo con mayor facilidad en un medio controlado. Autores como Michel et al. [32], Song [33] y Baena [35] realizan el aprovechamiento del bagazo de maguey verde (Agave salmiana) de la agroindustria mezcal en la producción de hongos ostra Pleurotus ostreatus, además de describir la importancia de mantener estas variables controladas en el proceso de producción, para así garantizar mejores condiciones en el desarrollo del cuerpo fructífero.

Al tener controladas las condiciones en el cultivo, se logra una adecuada invasión del micelio, el crecimiento de primordios y, por ende, el desarrollo del cuerpo fructífero, ya que el objetivo principal es lograr que el hongo se estrese e invada con mayor facilidad el sustrato, derivando así una reproducción del Pleurotus ostreatus en menor tiempo; lo cual representa ganancia económica para el cultivador y una disminución en el impacto ambiental, al utilizar un material vegetal de poco uso como es el caso de la cereza de café.

Tabla 2. Condiciones del cultivo para producción de hongos Pleurotus ostreatus.

\begin{tabular}{ccccc}
\hline \multirow{2}{*}{ Variables ( $\mathbf{\pm D}$. S) } & \multicolumn{5}{c}{ Producción de hongos } \\
\cline { 2 - 5 } & $\begin{array}{c}\text { Primera } \\
\text { cosecha }\end{array}$ & $\begin{array}{c}\text { Segunda } \\
\text { cosecha }\end{array}$ & $\begin{array}{c}\text { Tercera } \\
\text { cosecha }\end{array}$ & Promedio \\
\hline Humedad relativa $(\% \mathrm{HR})$ & $83,7 \pm$ & $73,9 \pm 8,5$ & $81,5 \pm$ & $79,7 \pm 5,1$ \\
& 4,6 & & $22,5 \pm$ & \\
Temperatura $\left({ }^{\circ} \mathrm{C}\right)$ & $22,4 \pm$ & & 0,9 & $22,8 \pm 0,6$ \\
& 1,6 & $23,5 \pm 0,9$ & 0,9 & $292,7 \pm$ \\
Luminosidad (Lux) & $293,2 \pm$ & $292,3 \pm$ & $292,6 \pm$ & 2037 \\
& 0,015 & 0,021 & 0,020 & 0,037 \\
\hline
\end{tabular}

( \pm D. S): desviación estándar.
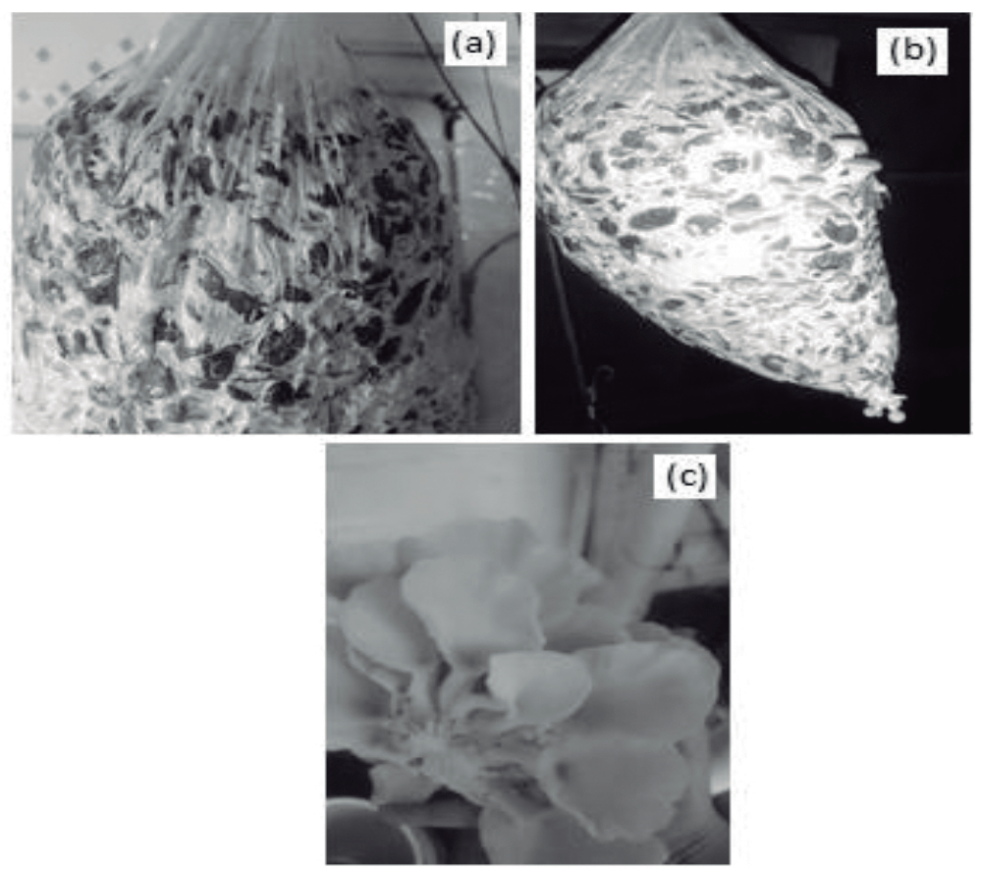

Figura 3. Obtención de hongos Pleurotus ostreatus en cereza de café. Donde A: Invasión de micelio, B: Aparición de primordios y C: Cuerpo fructífero. 
En la figura 3 se puede observar el desarrollo del cuerpo fructífero a partir del uso de la cereza de café como sustrato, mediante procesos biológicos como la degradación orgánica de esta biomasa; la figura (a) muestra la invasión óptima de micelio, mediante la aparición de la capa algodonosa producto de esa invasión. En la figura (b) se puede observar la formación de los primordios, la cual ocurrió a los 15 días después de la siembra del hongo, siendo superior al reportado por Alzate et al. [22] sobre sustratos de residuos orgánicos domésticos donde mencionan la aparición de primordios a los 12 días; eso se debe posiblemente a la calidad y capacidad que tenía la semilla del hongo de invadir el sustrato y al tamaño de partícula del material vegetal. Finalmente, en la figura (c) se observa el cuerpo fructífero a los 20 días, siendo similar a los valores reportados por Moran et al. [29], Mondragon et al. [34] y Baena [35] sobre sustratos de maíz y quínua, sustratos de bagazo de maguey verde (Agave salmiana) y sustratos de maíz, con la obtención del cuerpo fructífero entre 20 y 22 días.

El uso de esta biomasa permitió disminuir volúmenes de residuos y generar un subproducto de esta, generando una micorremediación; debido a los componentes con los que cuentan esos tipos de sustratos, como son los contenidos de lignina y celulosa del 28,954 $\pm 0,046$ y $48,390 \pm 0,326 \%$ respectivamente, entre otros componentes. Aquellos son consumidos por los hongos al realizar la descomposición aeróbica de un sustrato, generando un ambiente óptimo para su desarrollo, como lo indican Alzate et al. [22] y Miles y Chang [25]; se demostró que los hongos del género Pleurotus no solo secretan celulasa y hemicelulasas, sino que también producen enzimas que degradan la lignina, como lo describe Moran et al. [29], siendo una posible herramienta biotecnológica en el manejo de residuos agroindustriales. Al tener el cultivo del hongo sobre sustrato de cereza de café en un ambiente controlado y de fácil acceso, se pudo observar y determinar que la biomasa residual (cereza) de café se reduce cerca de un $80 \%$ de su peso inicial, esto debido a la capacidad con la que cuenta el sustrato de brindarle nutrientes y minerales al hongo y de este mismo para poder absorber y desarrollarse en este sustrato, siendo una alternativa viable de biorremediación; genera así una mayor productividad en menor cantidad de días, lo que se deriva en ganancias económicas para el agricultor y una disminución en contaminantes en el ambiente, además de la generación de un subproducto.

En cuanto a la producción de hongos Pleurotus ostreatus a partir de la biomasa residual cereza de café y su valor nutricional, se ha establecido por diferentes autores que es viable, pues estas setas es una fuente de fácil obtención de proteína; además, su producción es en corto tiempo y con una tecnología de poco requerimientos, lo que hace que el valor agregado aumente en el mercado, como lo mencionan Martínez y Nava [36], Garcia et al. [37], Rodríguez et al. [38] y Rivera et al. [39]. Estas setas son valoradas debido a su aroma y textura, ya que cerca del $2,5 \%$ de la población mundial las consuma anualmente.

De acuerdo a los resultados obtenidos en la caracterización fisicoquímica del material vegetal y al comparar estos con diferentes autores, se puede establecer que la biomasa residual que se ha utilizado para esta investigación (cereza de café) cuenta con las características físicas y sustancias químicas que se necesita para una posible producción del hongo Pleurotus ostreatus, teniendo como resultado una alternativa de biorremediación y una nueva oportunidad económica para los campesinos que quieran establecer este método de producción de hongos; cuenta además con una disminución del residuo en un 80 \% producto de la degradación por parte del hongo, esto debido a la capacidad que tiene el hongo de alimentarse principalmente de lignina y celulosa y de degradar la biomasa residual. Por lo anterior, es importante resaltar que esta investigación se puede establecer como una alternativa de biorremediación verde; ya que,se realiza una descomposición controlada de la biomasa residual y así, se protegen los diversos ecosistemas existentes en los límites de las fincas cafeteras, esto debido a la manera controlada con que se manejan los gases y los lixiviados que se producen en este proceso, como se establece en [8].

Finalmente, el poder tener una descomposición controlada de la biomasa residual cereza de café genera que se disminuya el impacto negativo que este produce en el ambiente, pues ya no se generaran gases que contribuyan al efecto invernadero y que por ende aumente la temperatura media del planeta [8], sino que también se tiene un control acerca de los lixiviados que se crean en el momento de descomposición, los cuales pueden ser ricos en metales pero que a su vez contaminen los cuerpos hídricos aledaños al espacio geográfico en donde se estén depositando [8]; también, el 
poder manipular y establecer diferentes criterios en el momento de obtener esta biomasa residual, genera que se disminuya la proliferación de insectos y roedores los cuales pueden afectar a los campesinos y los cultivos, pues la cereza de café ya no se estará depositando en las fincas cafeteras. Estas estrategias se fundamentan con el fin de fundamentar esta investigación como una alternativa verde de biorremediación.

\section{Conclusión}

La caracterización fisicoquímica de la biomasa residual (cereza) de café permitió determinar un porcentaje de lignina y celulosa del 28,954 $\pm 0,046$ y $48,390 \pm 0,326 \%$, respectivamente y materia orgánica del 13,249 $\pm 0,187 \%$, siendo adecuados para el debido crecimiento y desarrollo de los primordios y del hongo Pleurotus ostreatus, se observó se observó la aparición de los primordios a los 15 días y el hongo para cosechar a los 20 días, reduciendo el volumen de residuos en un $80 \%$. Es de suma importancia que estos residuos puedan ser utilizados para la generación de un nuevo producto y/o la disminución de este, lo que lograría un equilibrio entre el ambiente y los residuos que se generan, contribuyendo a la minimización del cambio climático.

\section{Agradecimientos}

Los autores agradecen a la dependencia de Investigaciones y a la Facultad de Ingenierías de la Universidad La Gran Colombia por el apoyo y acompañamiento durante la ejecución de esta investigación.

\section{Referencias bibliográficas}

[1] Banco Mundial. Agricultura, valor agregado (\% del PIB) (sitio de internet). Banco Mundial. Disponible en: https://datos.bancomundial. org/indicador/nv.agr.totl.zs. Acceso el 25 de febrero 2019.

[2] Cifuentes V. Durante 2018 la producción de café mundial fue de 168 millones de sacos de $60 \mathrm{~kg}$ (sitio de internet). La República. Disponible en: https://www.larepublica.co/ especiales/rutadel-cafe/durante-2018-laproduccion-de-cafemundial-fue-de-168-millones-de-sacos-de-60kg-2840566. Acceso el 25 de febrero 2019.

[3] Nieto J. Producción cafetera del país fue de 14,2 millones de sacos (sitio de internet). E
Tiempo. Disponible en: https://www.eltiempo. com/economia/sectores/produccion-de-cafeen-colombia-en-2017-167590. Acceso el 25 de febrero 2019.

[4] Instituto Geográfico Agustín Codazzi (IGAC). Cultivos predominan en la mitad del departamento de Quindío (sitio de internet). Instituto Geográfico Agustín Codazzi. Disponible en: https://www.igac.gov.co/es/ noticias/cultivos-predominan-en-la-mitad-deldepartamento-de-quindio. Acceso el 25 de febrero 2019.

[5] Suárez J. Aprovechamiento de los residuos provenientes del beneficio del café, en el municipio de Betania de Antioquia: Usos y aplicaciones (tesis maestría). Antioquia, Colombia: Corporación universitaria Lasallista; 2012.

[6] Del Toro Y. Obtención y caracterización de líquidos y carbón obtenidos por la pirolisis del aserrín de Acacia (Trabajo de pregrado). Cuba: Universidad de Oriente; 2012.

[7] Akhtar J, NorAishah A. A Reviews on operating parameters for optimum liquid oil yield in biomass pyrolysis. RSER. 2012;16(7):5101-9.

[8] Ballardares Grazzo C. Caracterización físicoquímica de los lixiviados del cacao y café del litoral ecuatoriano, como potenciales fuentes de producción de bioetanol (tesis de grado). España: Universidad de Las Palmas de Gran Canaria; 2016.

[9] Braham E, Bressani R. Pulpa de Café: Composición, tecnologíayutilización. Colombia: Centro Internacional de Investigaciones para el Desarrollo CIID; 1978.

[10] Rathinavelu R, Giorgio G. Posibles usos alternativos de los residuos y subproductos del café. (tesis de grado). Italia: Universidad de Trieste; 2005.

[11] Rodríguez C. Seminario internacional Gestión integral de residuos sólidos y peligrosos, siglo XXI (sitio de internet). Disponible en: http:// www.bvsde. paho.org/bvsacd/acodal/vi.pdf. Acceso el 20 de marzo 2019.

[12] Alvares Torres E. Contaminación ambiental generada por la emisión de gases (tesis de pregrado). Bogotá D.C, Colombia: Universidad Militar Nueva Granada; 2011.

[13] Norma AOAC. Método general del Codex para la determinación del pH. 981.12. Budapest: AOAC International; 2012.

[14] Norma AOAC. Official methods of analysis. Humedad en plantas. 20.013. USA: AOAC 
International; 1980.

[15] Norma NTC. Determinación del contenido de nitrógeno y cálculo del contenido de proteína cruda. Colombia: ICONTEC; 1999.

[16] Norma TAPPI. T 211 om-02 Ash in wood, pulp, paper and paperboard: combustion at $525^{\circ} \mathrm{C}$. USA: TAPPI; 2006.

[17] NMX-AA-021. Método para la determinación de materia orgánica en los residuos sólidos. México: Secretaría de Comercio y Fomento Industrial; 1985.

[18] Norma NTC. Determinación de contenido de grasa. Colombia: ICONTEC; 1972.

[19] Norma AOAC. Método Oficial 962.09. Fiber (Crude) in Animal Feed and Pet Food. USA: AOAC Internacional; 2002.

[20] Norma TAPPI. T222 Acid-insoluble lignin in wood and pulp. USA: TAPPI; 2002.

[21] Norma NTC. Pulpas para papel. Método para determinar las celulosas alfa, beta y gama. Colombia: ICONTEC; 1973.

[22] García L, Duque A, Padilla L, González L. Residuos orgánicos domésticos como sustrato para la producción de hongos Pleurotus ostreatus. Rev. Fac. Nal. Agr. 2014;67(2):11946.

[23] Rodríguez N, Araque L, Perdomo F. Producción de los hongos comestibles orellanas y shiitake. Colombia: Sección de Divulgación y Transferencia, Cenicafé; 2006.

[24] Gaitán R, Salmones R, Pérez M, Mata G. Manual práctico del cultivo de setas: aislamiento, siembra y producción (Tesis de grado). México: Instituto de Ecología, A.C.; 2006.

[25] Miles P, Chang ST. Biología de las setas. Fundamentos básicos y acontecimientos actuales. Colombia: Instituto ZERI para Latinoamérica; 1999.

[26] Grau C, Sánchez D, Zerpa A, García N. Influencia de la actividad del agua, $\mathrm{pH}$ y temperatura en el crecimiento de Aspergillus penicillioides y $A$. terreus aislados de la carne seca y salada de atún listado (Katsuwonus pelamis). Rev. Cient. 2007;17(2):193-9.

[27] Siguas B. Cenizas y Grasas (tesis de grado). Arequipa, Perú: Universidad Nacional De San Agustín; 2014.

[28] Docampo R. La importancia de la materia orgánica del suelo y su manejo en producción frutícola. Uruguay: Instituto Nacional de Investigación Agropecuaria- INIA; 2010.

[29] Moran F, Palazzi V, Sánchez M, Borsarelli
C. Combined UV-Vis absorbance and fluorescence properties of extracted humic substances-like for characterization of composting evolution of domestic solid wastes. Geoderma. 2009;11(3-4):61-7.

[30] Toledo M. Residuos de maíz y quinua como potenciales sustratos para el cultivo de hongos comestibles Pleurotus ostreatus (Trabajo de grado). Chimborazo, Ecuador: Escuela superior politécnica de Chimborazo; 2000.

[31]Blandón G, Dávila MT, Rodríguez N. Caracterización microbiológica y físico-química de la pulpa de café sola y con mucílago, en proceso de lombricompostaje. Rev. Cen. Café. 1999;50(1):5-23.

[32] Michel A, Otero M, Diaz E. Manual producción de hongos comestibles Pleurotus spp (sitio de internet). Disponible en: www. fundacionproducegro.org. mx/hongoscomestibles/. Acceso el 20 de diciembre 2018.

[33] Song B. Manual del cultivador de Hongos, cultivo del hongo ostra. Corea del Sur: MushWorld; 2005.

[34] Mondragon JM, Serna JA, García LS, Jaramillo LM. Caracterización fisicoquímica de los subproductos cáscara y vástago del plátano Dominico harton. rev. ion. 2017;30(2):21-4.

[35]Baena A. Aprovechamiento del bagazo de maguey verde (Agave salmiana) de la agroindustria mezcal en San Luis Potosí para producción de hongos ostra (Pleurotus ostreatus) (tesis de grado). México: Instituto Potosino de investigación científica y tecnológica; 2005.

[36] Martínez D, Nava D. Programa estratégico para el desarrollo de la producción, transformación y comercialización de hongos comestibles en el estado de Tlaxcala (tesis de grado). Tlaxcala A.C México: Colegio de Postgraduados en Ciencias Agrícolas y Fundación Produce; 2003.

[37] Garcia-Oduardo N, Bermúdez Savon RC, Serrano-Alberni M. Fórmula de sustratos en la producción de setas comestibles pleurotus. Tecnol. Quim. 2011;31(3):272-82.

[38] Rodríguez Valencia N, Araque Fonseca ML, Perdomo Perdomo F. Fructificación de hongos comestibles y medicinales. Colombia: FNC Cenicafé; 2006.

[39] Rivera RL, Martínez CA, Morales S. Evaluación de residuos agrícolas como sustrato para la producción de Pleurotus ostreatus. Luna Azul. 2013;37:89-100. 